\title{
Hunk/Mak-v is a negative regulator of intestinal cell proliferation
}

\author{
Karen R Reed ${ }^{1 * \dagger}$, Igor V Korobko ${ }^{2,3+}$, Natalia Ninkina ${ }^{2,4}$, Elena V Korobko ${ }^{3}$, Ben R Hopkins ${ }^{2}$, James L Platt ${ }^{2}$, \\ Vladimir Buchman ${ }^{2}$ and Alan R Clarke ${ }^{1}$
}

\begin{abstract}
Background: Conditional deletion of the tumour suppressor gene Apc within the murine intestine results in acute Wnt signalling activation. The associated over-expression of a myriad of Wnt signalling target genes yields phenotypic alterations that encompass many of the hallmarks of neoplasia. Previous transcriptomic analysis aimed at identifying genes that potentially play an important role in this process, inferred the Hormonally upregulated Neu-associated kinase (HUNK/Mak-V/Bstk1) gene as a possible candidate. Hunk is a SNF1 (sucrose non fermenting 1)-related serine/threonine kinase with a proposed association with many different tumour types, including colorectal cancer.
\end{abstract}

Methods: Here we describe the generation of a novel Hunk kinase deficient mouse which has been used to investigate the involvement of Hunk-kinase activity in intestinal homeostasis and tumourigenesis.

Results: We show that in the morphologically normal intestine, Hunk-kinase negatively regulates epithelial cell proliferation. However, the increase in cell proliferation observed in the Hunk kinase deficient intestine is counteracted by increased cell migration, thereby maintaining intestinal homeostasis. Using GRT-PCR, we further demonstrate that Hunk is significantly over-expressed in Apc deficient / Wnt-signalling activated intestinal tissue. Using the classical intestinal tumourigenesis $\mathrm{Ap} \mathrm{C}^{\mathrm{Min}}$ mouse model we show that loss of Hunk-kinase activity significantly reduced tumour initiation rates in the small intestine. However, an accompanying increase in the size of the tumours counteracts the impact this has on overall tumour burden or subsequently survival.

Conclusions: In the intestinal setting we demonstrate that Hunk has a role in normal intestinal proliferation and homeostasis and, although it does not alter overall survival rates, activity of this kinase does impact on tumour initiation rates during the early stages in tumourigenesis in the small intestine.

Keywords: Hunk, Mak-V, Intestine, Wnt signalling, Cancer, Apc ${ }^{\text {Min }}$ microarray

\section{Background}

Activation of the Wnt signalling pathway is a recognised early event in many intestinal cancers. Mouse models of intestinal neoplasia have proven to be invaluable in increasing our knowledge and understanding relating to the contribution of individual genes in this process. We have previously used Cre-Lox technology to conditionally delete the $A p c$ gene in the mouse intestine and characterised the phenotypic and transcriptional changes that occur following the acute activation of Wnt signalling in

\footnotetext{
* Correspondence: Reedkr@cf.ac.uk

${ }^{\dagger}$ Equal contributors

'University of Cardiff, European Cancer Stem Cell Research Institute, School of Biosciences, Cardiff CF10 3AX, UK

Full list of author information is available at the end of the article
}

this tissue [1]. Our microarray analysis demonstrated transcriptional activation of the Hormonally upregulated Neuassociated kinase (HUNK/Mak-v/Bstk1) gene immediately following Apc loss, indicating that Hunk is potentially a Wnt signalling target gene which could play a role during the initial stages of intestinal neoplasia. Hunk is a SNF1 (sucrose non fermenting 1)-related serine/ threonine kinase that was originally cloned by Korobko et al. $[2,3]$ and Gardner et al. [4] but its function still remains largely unknown. A variety of binding partners for Hunk have been identified including Nedd4 E3 ubiquitin ligase [5], Synaptopodin [6], Rabaptin-5 [7] and cofilin-1 [8], although the molecular mechanisms of Hunk action remain unclear. Hunk has been shown to be expressed in a variety 
of tissues but is most notably associated with pregnancyinduced alterations in the mammary gland and high levels of expression within the brain $[4,9]$. Two independent studies have shown that Hunk is able to negatively regulate proliferation in normal epithelial cells. Gainof-function and loss-of-function studies within mouse distal convoluted tubule (mDCT) cells, demonstrated that Hunk negatively regulates ANG II-induced c-fos gene expression and $\mathrm{mDCT}$ proliferation [10]. Furthermore, MMTV-driven Hunk over-expression within mammary epithelium, inhibits proliferation of alveolar epithelial cells during mid-pregnancy [9]. However, within the cancer setting, both pro- and antitumourigenic properties for Hunk have been described. Overexpression of Hunk has been shown in a number of different cancers, and it is thought to be associated with the more aggressive subset of carcinomas $[11,12]$, probably due to its ability to support cell viability and survival $[3,13,14]$. Using transgenic mouse models, Yeh et al. [13] have shown that Hunk plays a role in tumour initiation and is required to facilitate HER2/neu-induced mammary tumourigensis. Contrary to this, Wertheim et al. [12] demonstrated that Hunk was dispensable for tumour initiation in a MMTV-cMyc driven model of mammary tumourigenesis, but was essential for tumour metastasis, and therefore impacted on overall survival in this mouse tumour model. Both of these studies suggest Hunk functions in a pro-tumourigenic manner. Conversely, in a xenograft model of mammary tumourigenesis using a basal breast cancer cell line in which Hunk was over expressed, Quintela-Fandino et al. [8] demonstrate that Hunk overexpression suppresses metastasis, suggesting a tumour suppressor role for Hunk. However, the differences in the experimental setup of these studies make it difficult to draw any firm conclusions as to the role of Hunk in tumourigenesis. Although over-expression of Hunk has been shown to be associated with advanced and aggressive forms of carcinoma [12], no one to date has studied the importance of Hunk in intestinal tumourigenesis. Indeed, analysis of the Oncomine database confirmed the association of Hunk expression and intestinal cancer. For breast cancer, the cancer conventionally associated with Hunk, 1 out of 27 analyses (3.7\%) within the Oncomine database demonstrate greater than 1.5 fold over-expression of Hunk ( $<<0.01$ ). However, using the same cut-off criteria, 5 out of 24 analyses (28.8\%) associated with colorectal cancer. This clearly indicates that overexpression of Hunk is potentially important in intestinal cancer and warrants further investigation.

Loss of the tumour suppressor protein adenomatosis polyposis coli (APC) and activation of the Wnt signalling pathway is recognised as an early key event in the majority of intestinal neoplasia. Work within Xenopus embryos has demonstrated that Hunk has the ability to modulate Wnt signalling, which is presumed to be via Hunk directed phosphorylation of Disheveled [15]. Here we describe the generation of a novel Hunk-kinase deficient mouse, and the subsequent investigation of the contribution of Hunk-kinase in normal intestinal homeostasis and tumourigenesis using this novel knock-out.

\section{Methods}

\section{Targeting construct}

A plasmid construct for targeting exon 4 of the mouse Hunk gene (Figure 1D) was generated using pPNT vector [16] as a backbone with $0.95 \mathrm{~kb}$ "short arm" cloned into EcoRI site and $4.84 \mathrm{~kb}$ "long arm" cloned between XhoI and NotI sites. "Short" and "long arms" were obtained by PCR amplification of E14Tg2a embryonic stem (ES) cell genomic DNA with high-fidelity Platinum Pfx or AccuPrime polymerases (Invitrogen) and specific primers designed on the basis of $\mathrm{C} 57 \mathrm{Bl} / 6 \mathrm{~J}$ genome sequence (GeneBank Acc.No. NT 039625 2).

\section{Targeting Hunk gene in ES cells}

E14Tg2a mouse ES cells were used to target Hunk allele as described in our previous publications [17]. Briefly, ES cells were transfected with NotI-linearised targeting plasmid by electroporation and clones were selected with G418 positive and gancyclovir negative selections. Clones were screened by PCR on genomic DNA template with primers MK1S 5' -tgagttgagggcttggtgttctttg-3' located upstream of the "short arm" and neoB 5' -aagaacgagatcagc agcc-3' located inside neomycin phosphotransferase expression sequence. Clones with homologous recombination of the "short arm" were identified by amplification of $1.1 \mathrm{~kb}$ fragment (Figure 1D). Homologous recombination of the "long arm" was analyzed by Southern blot analysis of genomic DNA digested with BamHI with P32-labeled probe " $\mathrm{L}$ ". While digestion of the wild type allele results in $10.3 \mathrm{~kb}$ fragment detected by hybridization, homologous recombination of the "long arm" lead to the appearance of an additional $9.3 \mathrm{~kb}$ fragment (Figure 1D).

\section{Generation of mice with targeted Hunk gene}

Successfully targeted ES cell clones with normal chromosome complement were used for generating mouse chimeras by blastocyst (C57Bl/6 J) injection. The germ-line transfer was assessed by breeding male mouse chimeras with $\mathrm{C} 57 \mathrm{Bl} / 6$ female mice. Presence of Hunk+and Hunk-alleles was confirmed by PCR with primers MKK (5'-tagtctggttggcatcaccg-3'), MK1A (5'-cagaatccagctag acctaacagtg-3') and neoB on templates of genomic DNA isolated from mouse tail biopsies. Amplification with primers MK1A and MKK on Hunk + allele resulted in 383 bp amplification product while PCR with neoB and MKK primers on Hunk allele resulted in amplification of a $476 \mathrm{bp}$ fragment (Figure 1D). PCR reaction 

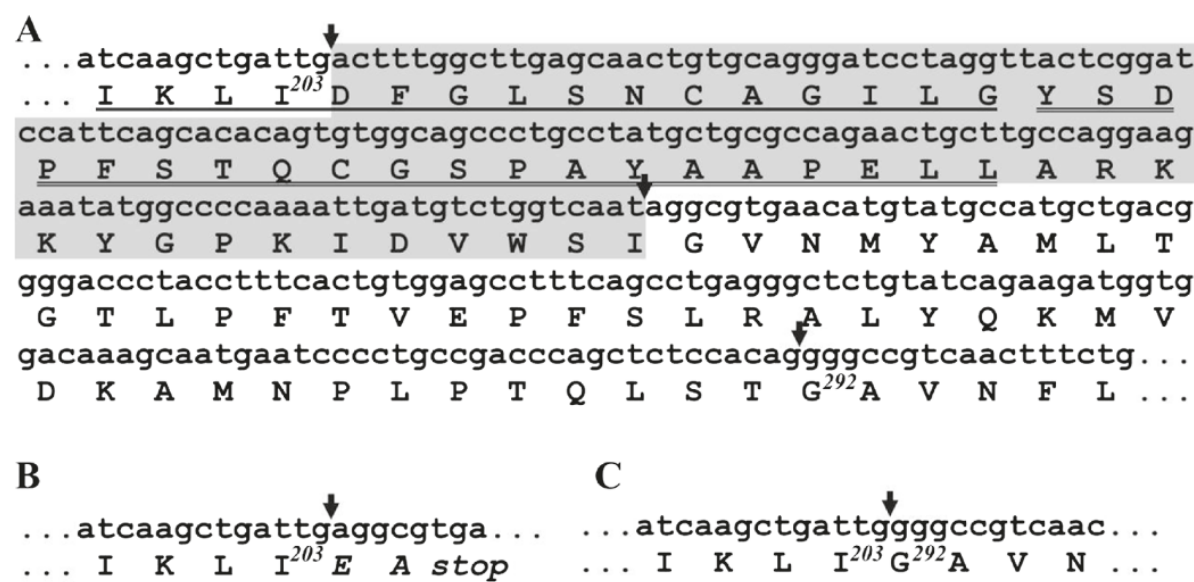

D

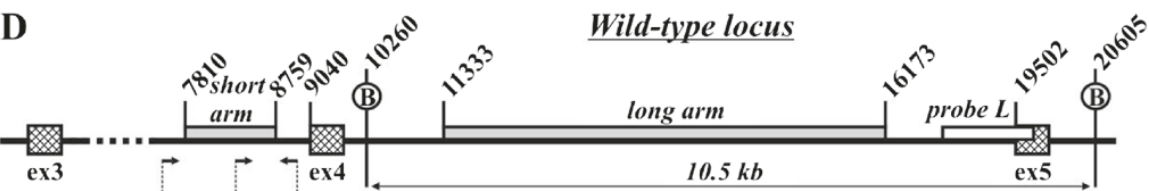

$\overrightarrow{\mathrm{ex} 3} \quad \overrightarrow{\mathrm{t}} \mathrm{ex4}$

$\begin{array}{lll}7780 & 8402 & 8785\end{array}$

MKIS MKK MKIA

$\underline{\text { Targeting construct }}$

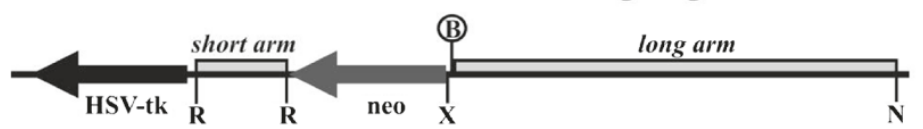

Targeted locus

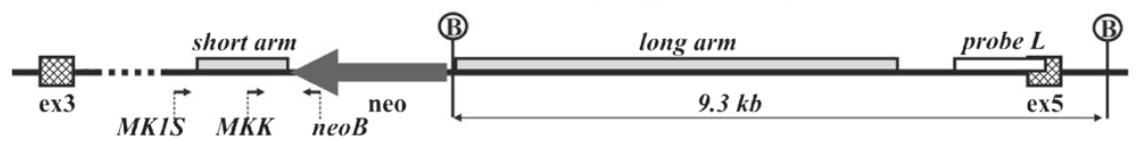

E
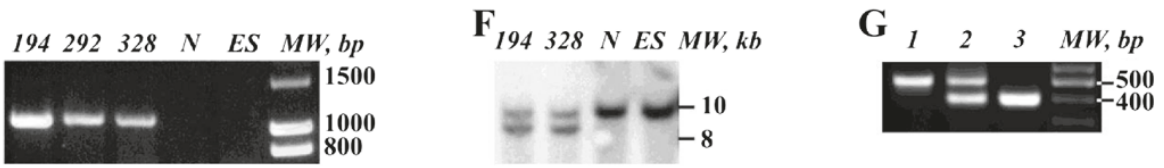

H

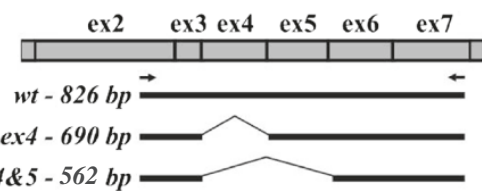

I

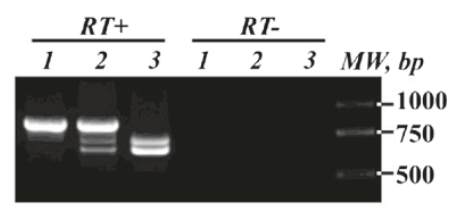

Figure 1 Strategy for targeting the Hunk allele. A) Nucleotide sequence of mouse Hunk cDNA corresponding to targeted exon 4 (highlighted). Arrows mark exon/intron junctions. Subdomains VII (single-underline) and VIII (double-underline) of the catalytic domain are indicated. B, C Nucleotide sequences of Hunk cDNA with spliced out exon 4 (B) or exons 4 and 5 (C). Note, a frame shift and truncation protein truncation occurs following splicing between exons 3 and 5. However, splicing between exons 3 and 6 (which doesn't induce a frame shift), produces a protein lacking subdomains important for kinase activity. D Schematic of the targeting strategy. Hatched boxes represent exons. EcoRl (R), Xhol (X), Notl ( $M$ ) sites (for cloning "short" and "long arms" into pPNT vector) and BamHI (B) sites (for "long arm" recombination analysis) are shown. Arrows represent primers (used for ES clone analysis and genotyping of mice). Nucleotide positions are shown according to mouse chromosome 16 sequence GeneBank Acc. No. NT 039625 2. Neomycin phopshotransferase (dark grey arrow) and HSV thymidine kinase (black arrow) expression cassettes, are also shown. E PCR analysis of ES clones (primers MK1S and neoB) demonstrating homologous recombination of the "short arm" in clones 194, 292 and 328 but not in negative clone N or parental E14Tg2a ES cells (ES). F Southern blot analysis using P32-labeled probe "L" (white box in panel D) and BamHI-digested genomic DNA from clones 194 and 328, negative clone N and parental E14Tg2a ES cells (ES). G PCR genotyping of Hunk-/-(lane1), Hunk+/-(lane2) and Hunk+/+(lane3) mice (primers MK1A, MKK and neoB). $\mathbf{H}$ Scheme of exons 2 through 7 of Hunk CDNA, demonstrating amplification products from wild type allele (wt), targeted allele missing exon 4 (KODex4) and with additionally spliced out exon 5 (KODex4\&5). Arrows represent RT-PCR Primers. I RT-PCR amplification from cerebellum RNA of Hunk+/+(lane1), Hunk+/-(lane2) and Hunk-/-(lane3) mice. MW - DNA molecular weight markers. 
contained $1 \mathrm{mM} \mathrm{MgCl} 2$ in Taq DNA polymerase reaction buffer, $0.2 \mathrm{mM}$ dNTP, $2 \mathrm{U}$ of Taq DNA polymerase (Fermentas), primers MKK, MK1A, neoB and genomic DNA template in the final volume of $25 \mu \mathrm{l} .35$ cycles of $15 \mathrm{sec}$ at $94^{\circ} \mathrm{C}, 30 \mathrm{sec}$ at $63^{\circ} \mathrm{C}$, and $60 \mathrm{sec}$ at $72^{\circ} \mathrm{C}$ were carried out on DNA EngineDyad amplifier (BioRad).

\section{Mice and sample preparation}

This study received ethical approval from Cardiff University's Animal Welfare and Ethical Review Body (previously known as the ERP), and all animal procedures were conducted in accordance with UK Home Office regulations. AhCre $+A p c+/+$ and $\mathrm{AhCre}+\mathrm{Apcfl} / f l$ mice were generated and maintained on an outbred background as previously described [1]. Cre-recombinase activity was induced from the Ah-Cre transgene by three intraperitoneal (IP) injections of $80 \mathrm{mg} / \mathrm{kg} \beta$-naphthoflavone within $24 \mathrm{~h}$. Mice were sacrificed at day 4 . Cohorts containing the $A p c^{M i n}$ allele were sacrificed when animals displayed symptoms of intestinal disease, including weight loss, rectal bleeding and criteria of anaemia (as assessed by pale feet). Tissues were harvested, fixed and processed according to standard protocols as previously described [1].

\section{BrdU labelling}

To achieve BrdU labelling for proliferation and migration studies, mice were administered with $250 \mu \mathrm{l} \mathrm{BrdU}$ (Amersham) via an IP injection either $2 \mathrm{hrs}$ or $24 \mathrm{hrs}$ prior to culling ( $\mathrm{n}=3$ in all cases). Immunohistochemical (IHC) staining for BrdU was performed using an antiBrdU antibody (BD biosciences 1:500). BrdU-positive cell position and number were scored. Kolmogorov-Smirnov test proved a significant difference between the distributions of BrdU-positive epithelial cells in crypts, $24 \mathrm{hr}$ post BrdU administration.

\section{RT-PCR analysis}

Total RNA extraction and first-strand cDNA synthesis were carried out as described previously [18]. For analysis of Hunk expression in mouse tissues one $\mu \mathrm{l}$ of cDNA was used as a template for PCR amplification with primers $5^{\prime}$ agatccagcagatgatccgac- $3^{\prime}$ and $5^{\prime}$-tagcgctcaagtttcttgttcaa- $3^{\prime}$ and Platinum AccuPrime DNA polymerase (Invitrogen). 35 cycles of $15 \mathrm{sec}$ at $95^{\circ} \mathrm{C}$ and $90 \mathrm{sec}$ at $68^{\circ} \mathrm{C}$ were carried out on DNA Engine Dyad amplifier (BioRad). qPCR was performed using Applied Biosystems TaqMan Universal PCR mix and Steponeplus machine. The $2-\Delta \Delta C T$ method [19] was used to calculate relative fold change in expression levels, with $\beta$-Actin expression being used as the housekeeping gene, which we can confirm amplified with an equivalent efficiency to the test primers. The mean $\Delta C T$ values for the experimental groups were compared to the mean $\Delta \mathrm{CT}$ values for the control group, in order to provide the relative fold change. Thus, figures representing relative fold change do not possess error bars, although statistical significance between the $\Delta C T$ values was tested using the Mann-Whitney $U$ test and deemed significant when $\mathrm{p}<0.05$. Primers used were: Hunk 5'atcacacagctccagagtacca3' and 5'ggttggtgtggctcta gtttct3', $\beta$-actin 5'caccacaccttctacaatgagc3' and 5'gtacga ccagaggcatacagg3', Axin2 5'gcagctcagcaaaaagggaaat3' and 5'tacatggggagcactgtctcgt3', Wif1 5'aacaagtgccagtgtcgaga gg3' and 5' gcctttttaagtgaaggcgtgtg3'.

\section{Affymetrix microarray analysis}

Normal colonic and paired polyp tissue was collected from symptomatic $A p c^{M i n}$ mice. Biotinylated target cRNA was generated from these tissues as previously described $[1,20]$. Affymetrix MOE430_2 gene arrays were run at the CRUK facility at the Paterson Institute for Cancer Research, and the data has been deposited in NCBI's Gene Expression Omnibus and are accessible through GEO Series accession number GSE65461 (http://www.ncbi.nlm. nih.gov/geo/query/acc.cgi?acc=GSE65461). Arrays from AhCre $+A p c+/+$ and AhCre + Apcfl/fl mice have previously been published for intestinal tissue [1] and liver tissues [20].

\section{Results}

\section{Wnt signalling activation results in up-regulated Hunk} expression

Apc is a known key regulator of Wnt signalling, and critically important in regulating normal intestinal homeostasis. Conditional deletion of $A p c$ within the mouse intestine using an Ah-Cre recombinase to drive recombination of LoxP flanked $A p c$ alleles, has previously been shown to result in acute activation of Wnt signalling and many hallmarks of neoplasia, including increased proliferation and apoptosis and loss of differentiation and migration [1]. Affymetrix microarray analysis indicates an acute transcriptional activation of Hunk following the loss of $A p c$ in the intestine and liver and in colonic adenomas from the $A p c^{\mathrm{Min}}$ mouse (Figure 2). qRT-PCR analysis confirms the transcriptional activation of Hunk in these settings (Figure 1), indicating that Hunk transcription is coincident with Wnt signalling activation and tumour formation. Indeed, a Tcf/LEF consensus binding site can be found within the promoter region of Hunk, and significant up-regulation of HUNK has been shown to occur in human colorectal cancer cell lines [12].

\section{Hunk-kinase deficiency results in increased intestinal cell proliferation}

To assist in our quest to investigate the importance of Hunk-kinase in intestinal tumourigenesis, we generated a novel mouse line carrying a Hunk-kinase deficient allele. To do this exon 4 of the mouse Hunk gene was targeted. 


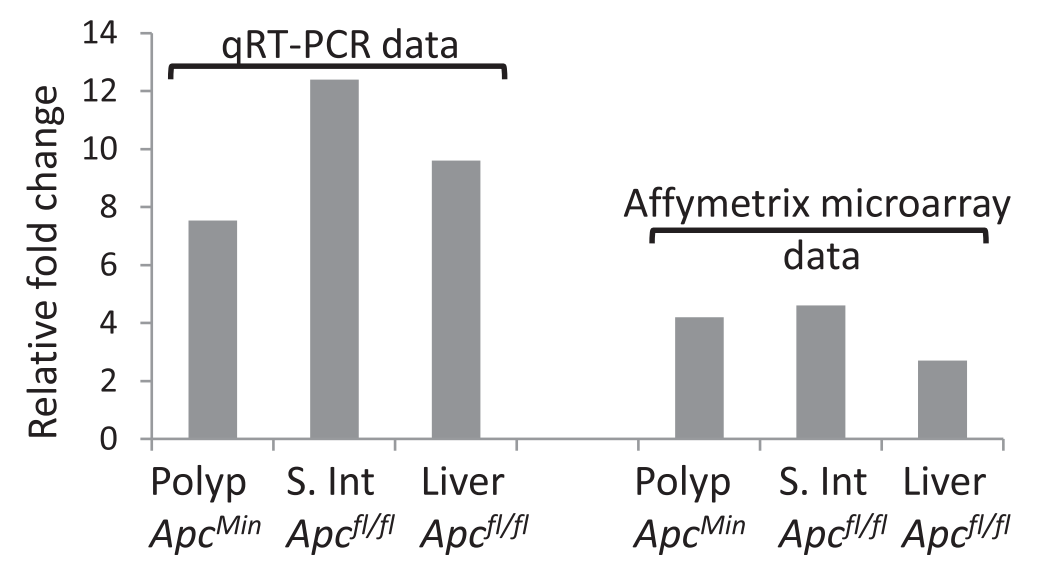

Figure 2 A Relative fold change in the levels of Hunk expression compared to the appropriate normal tissue assessed using qRT-PCR analysis and Affymetrix micro-array (Intestinal array [1], liver array [20]). In all cases Mann-Whitney $U$ test reveals a significant difference between $\Delta C T$ values $(p<0.05)$. Small intestine $(S$. Int) and liver samples were collected Day 4 post Ah-Cre induction. Normal colonic tissue and adjacent adenoma tissue (polyp) were taken from $\mathrm{ApC}^{\mathrm{Min}}$ animals.

A protein fragment encoded by this exon (amino acids 204-249) contains a part of subdomain VII (starting from conserved Asp204 which is important for $\gamma$-phosphate of MgATP orientation), the entire subdomain VIII, which is critical for substrate recognition, and a portion of subdomain IX of the Hunk protein kinase catalytic domain. Therefore, deletion of exon 4 results in the production of a catalytically inactive Hunk protein. Moreover, deletion of the exon 4 results in a shift of the open reading frame in the transcript after joining exons 3 and 5 . As a result, the translated protein would consist of only 203 amino acids of the Hunk polypeptide with translation terminating 2 codons downstream of the codon encoding Ile203 (Figure 1A and B). Following successful targeting in ES cells (Figure 1D, F) and the production of chimeric animals after ES cell injection into blastocysts, successful germ line transmission of the targeted allele was confirmed by PCR (Figure 1G). Transgenic mice were further back-crossed for 6 generation to obtain a mouse line on a pure $\mathrm{C} 57 \mathrm{Bl} / 6$ J genetic background.

Due to the lack of suitable antibodies for the detection of endogenous Hunk protein in mouse tissue, RT-PCR was used to analyze Hunk transcripts in wild type, Hunkheterozygous and homozygous mice (Figure $1 \mathrm{H}, \mathrm{I})$. The Hunk+/+ yielded the expected 826 bp PCR fragment corresponding to the wild type Hunk allele, while it was completely absent in samples of Hunk-/- animals (Figure 1I). However, along with a $690 \mathrm{bp} \mathrm{PCR} \mathrm{fragment}$ corresponding to mRNA lacking exon 4 , an additional prominent amplification product, a 562 bp fragment, was detected in Hunk-/- animals (Figure 1I). Cloning and sequencing revealed that this fragment represents a Hunk transcript lacking not only targeted exon 4 but also exon 5 sequences. Importantly, deletion of both exons 4 and 5 (Figure $1 \mathrm{H}$ ), while resulting in transcript encoding catalytically inactive protein due to deletion of catalytic domain portion, does not lead to a frame-shift and the translated protein should be identical to full-length Hunk except for the deletion of amino acids 204-291 (Figure 1C). In heterozygous Hunk+/- mice, the wild type allele transcript was substantially more abundant than both variants of the mutant allele transcript (Figure 1I).

Given our interests in the role of Hunk in intestinal tumourigenesis, a detailed examination of the phenotype in Hunk-/- intestine was performed. No differences were found in the representation of the different cell types of the intestine (assessed using alcian blue staining for goblet cells, lysozyme IHC for paneth cells and grimelius staining for enteroendocrine cells), suggesting Hunk-kinase activity is not involved in lineage specification in the intestine (Figure 3A). However, although the gross morphology remained unaltered, with the number of cells within the crypt remaining the same, Hunk-/intestine displayed a significant increase in crypt cell proliferation within the small intestines (scored using BrdU incorporation and histological examination of intestinal crypts, Figure 3B). This was not accompanied by any alteration in the rates of apoptosis (Figure 3B), although migration rates along the crypt-villus axis were significantly perturbed; Hunk-/- intestinal cells display an increased rate of migration (Figure 3C). Consequently, these data demonstrate that within a normal intestinal setting, loss of kinase active Hunk was sufficient to induce alterations in the normal intestinal kinetics, but this did not alter normal intestinal physiology.

\section{Hunk-kinase deficiency alters tumour initiation rates but} not survival in $A p c^{M i n}$ mice

In order to address the importance of Hunk-kinase in Wnt driven intestinal tumourigenesis, the Hunk-kinase 


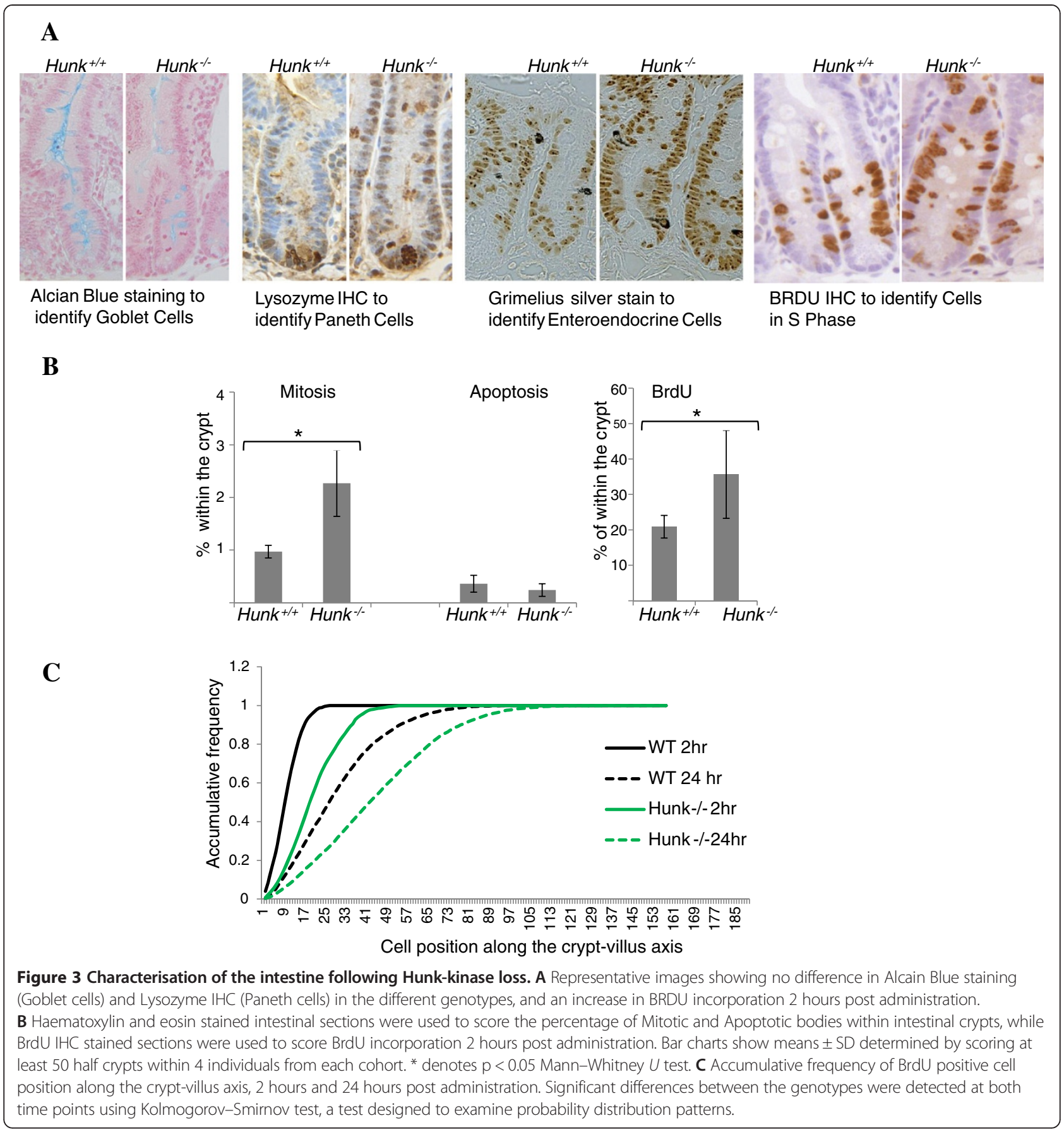

deficient mice were inter-crossed with the established $A p c^{M i n}$ mouse model of intestinal cancer. Cohorts of $\mathrm{Apc}^{\mathrm{Min}} \mathrm{Hunk}^{+/+}, \mathrm{Apc}^{\mathrm{Min}} \mathrm{Hunk}^{+/-}$and $\mathrm{Apc}^{\mathrm{Min}} \mathrm{Hunk}^{-/-}$littermates were generated and aged and monitored until the animals displayed overt symptoms of intestinal disease, at which stage they were culled using the appropriate schedule 1 method, and tissues were harvested. Kaplan-Meier survival analysis demonstrated that loss of kinase active Hunk does not alter the survival of $A p c^{\mathrm{Min}}$ mice (Figure 4A). However, macroscopic scoring of tumours at dissection showed significantly fewer $(\mathrm{p}=0.025$ Mann-Whitney), yet larger tumours in the $\mathrm{ApC}^{\mathrm{Min}} \mathrm{Hunk}^{-1-}$ cohort (mean number 21.4per animal +/- 2.7SEM, mean size $7.1 \mathrm{~mm} 2+/-0.3 \mathrm{SEM})$ compared to the $A p c^{\mathrm{Min}}$ Hunk $^{+/+}$cohort (mean number 36.2 per animal +/4.8SEM, mean size $5.7 \mathrm{~mm} 2+/-0.3 \mathrm{SEM}$ ). Furthermore, this difference was restricted to the small intestine (Figure 4B). Detailed microscopic analysis of these tumours did not reveal any differences in the stage, types or characteristics of the tumours occurring in the different cohorts. 


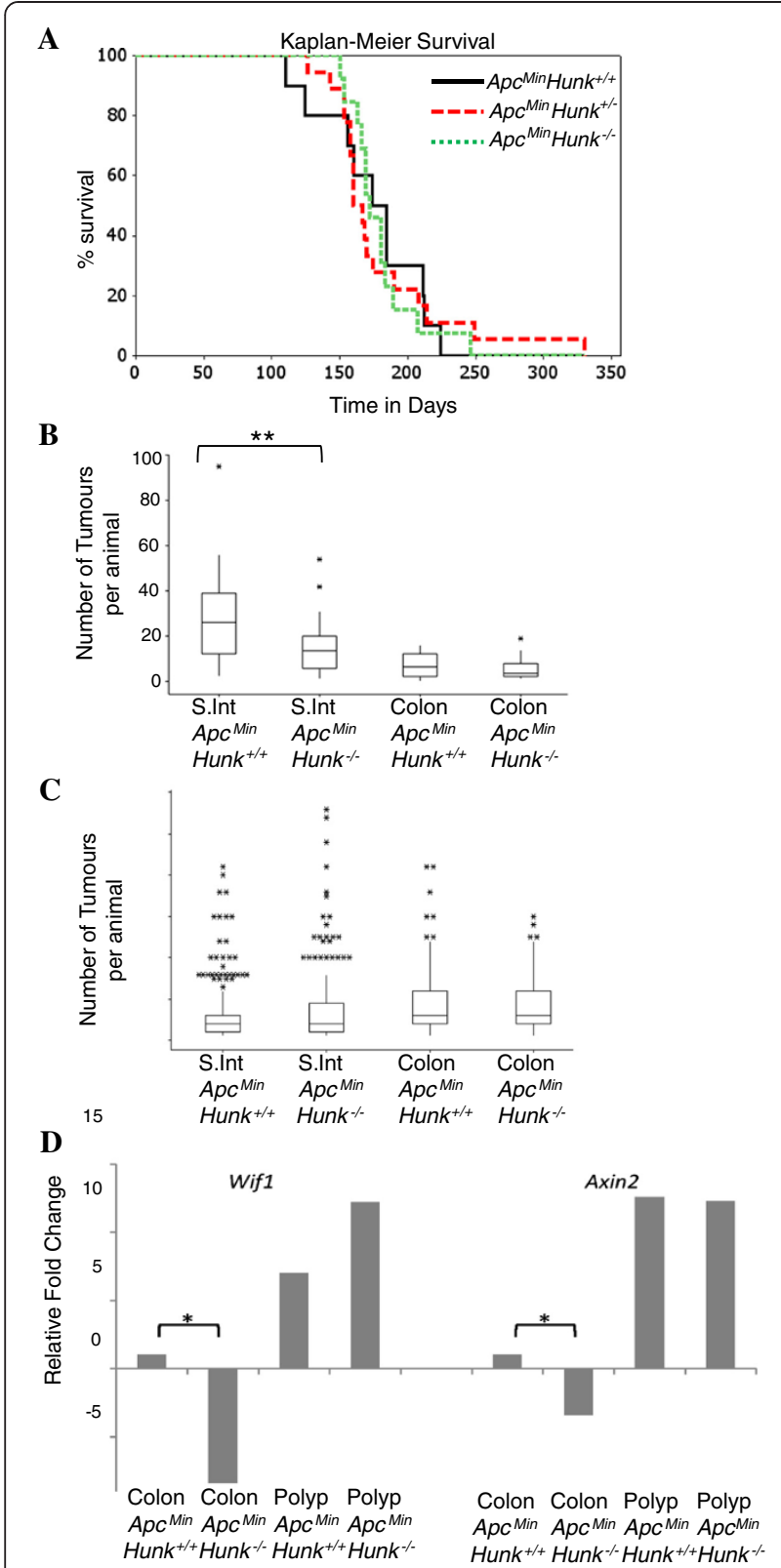

Figure 4 Survival and tumour burden analysis. A Kaplan-Meier

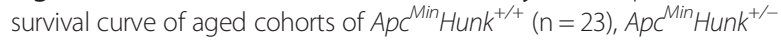
$(\mathrm{n}=27)$ and $\mathrm{ApC}^{\mathrm{Min}} \mathrm{Hunk}^{-/-}(\mathrm{n}=23)$ mice, demonstrating that no significant differences in survival between the cohorts. B Box plot displaying the total number of tumours found at death in the aged cohorts of $\mathrm{ApC}^{\mathrm{Min}} \mathrm{Hunk}^{+/+}$and $\mathrm{ApC}^{\text {Min Hunk }}{ }^{-/-}$mice. The box encompasses the first quartile (at bottom) to the third quartile (at top) of the data set and the horizontal boxed line represents the median. ${ }^{* *} p<0.01$ Mann-Whitney $U$ test. C Box plot displaying the size of tumours found at death in the aged cohorts of $\mathrm{ApC}^{\text {Min }} \mathrm{Hunk}^{+/+}$ and $\mathrm{ApC}^{\mathrm{Min}} \mathrm{Hunk}^{-/-}$mice. $\mathbf{D}$ qRT-PCR analysis showing relative expression levels of Axin2 and Wif1 in normal colonic tissue (colon) and adjacent adenoma tissue (polyp) taken from 4 aged matched animals from the different genotypes. ${ }^{*} p<0.05$ Mann-Whitney $U$ test between $\Delta C T$ values.
Neither the proliferation rates nor apoptosis rates within tumours differed with Hunk-kinase status (Additional file 1: Figure S1). Furthermore, qRT-PCR analysis for Wnt target genes (including $c M y c$, Ascl2, Axin2, CD44, CD1, Sox17, Wif1 and Tiam1) did not identify any significant differences between the colonic tumours isolated from both cohorts of mice. However, it is pertinent to note that both Wif1 and Axin2 were significantly down-regulated within the normal colonic Apc ${ }^{\mathrm{Min}} \mathrm{Hunk}^{-/-}$tissue compared to normal colon samples from $\mathrm{Apc}^{\mathrm{Min}} \mathrm{Hunk}^{+/+}$mice (Figure 4D). Thus, kinase active Hunk positively contributes toward the normal expression of two negative regulators of canonical Wnt signalling within normal intestinal tissue, but loss of Hunk-kinase and the subsequent down regulation of Axin 2 and Wif1 does not confer a generic mis-regulation of Wnt signalling. A precise molecular characterisation following the loss of Hunk-kinase is required to ascertain the exact mechanism by which this kinase influences gene transcription and cell proliferation. Overall, in relation to intestinal tumourigenesis our results show that Hunk-kinase activity does impact on intestinal tumour initiation within the small intestine, but this is not sufficient to alter the overall tumour burden or survival in the $A p c^{M i n}$ mouse model of intestinal cancer.

\section{Discussion}

The exact role of the SNF1-related serine/threonine kinase Hunk (Mak-V) remains unclear. Here we have generated a novel kinase-deficient Hunk allele, and produced homozygous Hunk-kinase deficient mice, Hunk-/-. Two previous studies have shown Hunk to be a negative regulator of proliferation within normal epithelial cells $[9,10]$ and our findings support this notion. We have shown that loss of Hunk-kinase activity within the normal intestinal setting results in an increase in proliferation within epithelial cells. This is accompanied by an increase in cell migration rates, thereby maintaining normal physiology despite altered kinetics.

An increasing body of evidence links the function of Hunk with cancer initiation, progression and metastasis [3,8,11-13], although there remains uncertainty regarding the precise involvement of Hunk in tumourigenesis. To date, most studies have analysed the role of Hunk in mammary tumourigenesis, largely due to the known role of this protein in mammary gland development [4]. However, our microarray findings implicated Hunk in intestinal tumourigenesis, a role we wished to elucidate further. We have shown that Hunk expression becomes significantly up-regulated from the earliest stages of tumour initiation following $A p c$ loss, indicating this gene is probably a Wnt signalling target gene. Indeed a Tcf/LEF binding motif can be found within the promoter region of Hunk. We appreciate this evidence is circumstantial, and a 
more detailed interrogation is required to confirm Hunk as a Wnt target gene.

Studies using Xenopus embryos have shown that Hunk has the ability to modulate Wnt signalling. We used qRT-PCR analysis to examine this in the intestine and demonstrated a significant reduction in expression levels of two negative regulators of Wnt signalling, Wif1 and Axin2, accompanying the loss of kinase active Hunk. However, this did not translate to a generic de-regulation of Wnt signalling. Interestingly, contrary to a recent publication by Yeh et al. [14] who described Hunk as a negative regulator of cMyc expression, we did not observe any significant alteration in the levels of cMyc transcription accompanying Hunk-kinase loss. Discrepancies in the examined tissues and experimental setup might account for these differences. In an attempt to explain the mis-regulation of both Wif1 and Axin2 which can be regulated by components of the BMP/ TGFßpathway [21,22], qRT-PCR analysis of components and targets of this pathway was performed. However once more, a generic mis-regulation of this pathway was not confirmed by qRT-PCR analysis. A more detailed genomic wide study would be required to confidently identify the mechanism through which Hunk-kinase is able to negatively regulate proliferation in the intestine.

Intercrossing Hunk-/- mice with $A p c^{M i n}$ mice allowed us to determine the role of Hunk-kinase in Wnt signalling driven intestinal tumourigenesis. We have shown a significant reduction in the tumour initiation rate within the small intestine in $A p c^{\mathrm{Min}} \mathrm{Hunk}^{-1-}$ mice, but this does not impact on overall survival due to an accompanying increase in the size of those tumours that do form. It is possible that the reduced tumour initiation rate is associated with the increased cell turnover rate along the crypt-villus axis (increased proliferation and migration) seen following the loss of Hunk-kinase, although the exact mechanisms for this have not been elucidated. Further studies would be required to determine the significance of these subtle changes associated with the lack of kinase active Hunk.

Overall, our data confirm Hunk-kinase as a negative regulator of normal epithelia proliferation, and demonstrate that in the classical $A p c^{\text {Min }}$ mouse model of intestinal tumourigenes, Hunk-kinase activity significantly impacts on tumour initiation rates during the early stages in tumourigenesis.

\section{Conclusions}

Here we describe the production of a new Hunk-kinase deficient mouse model and use it to examine the importance of this kinase during the early stages of intestinal tumourigenesis. We show that despite not affecting overall survival of the $A p c^{M i n}$ mice, Hunk-kinase is a negative regulator of normal intestinal proliferation, and impacts significantly on small intestinal tumour initiation rates.

\section{Availability of supporting data}

The Affymetrix array data has been deposited in NCBI's Gene Expression Omnibus and are accessible through GEO Series accession number GSE65461 (http://www. ncbi.nlm.nih.gov/geo/query/acc.cgi?acc=GSE65461).

\section{Additional file}

Additional file 1: Figure S1. Mitosis and apoptosis levels scored from $\mathrm{H}+\mathrm{E}$ stained sections on intestinal adenomas. Bar charts show means SD of values obtained from at least 6 tumours from three individuals within each cohort.

\section{Abbreviations}

APC: Adenomatosis polyposis coli; ES: Embryonic stem cell; HUNK: Hormonally upregulated Neu-associated kinase; IHC: Immunohistochemistry; IP: Intraperitoneal; MDCT: Mouse distal convoluted tubule; S. Int: Small intestine.

\section{Competing interests}

The authors declare that they have no competing interests.

\section{Authors' contributions}

KRR, IVK, VB and ARC designed the research, IVK, NN and EVK generated the novel Hunk- allele, KRR managed the mouse intercrosses, $\mathrm{BH}$ and JLP performed IHC and qRT-PCR, KRR analysed data. The manuscript was drafted by KRR and all authors critically reviewed. All authors read and approved the final manuscript.

\section{Acknowledgements}

This work was supported by a Cancer Research UK program grant awarded to ARC, a Wellcome Trust VIP award awarded to KRR, a Wellcome Trust Short Term Travel Grant 071269 and a INTAS Young scientist Fellowship awarded to IV, and Nuffield Bursary summer studentships awarded to JLP and BH. We thank Mark Bishop and Derek Scarborough for technical services and support with genotyping and histology.

\section{Author details}

${ }^{1}$ University of Cardiff, European Cancer Stem Cell Research Institute, School of Biosciences, Cardiff CF10 3AX, UK. ${ }^{2}$ School of Biosciences, University of Cardiff, Cardiff CF10 3AX, UK. ${ }^{3}$ Russian Academy of Sciences, Institute of Gene Biology, 34/5 Vavilov street, Moscow 119334, Russia Federation. ${ }^{4}$ Institute of General Pathology and Pathophysiology of Russian Academy of Medical Science, 8 Baltijskaya str, Moscow 125315, Russian Federation.

Received: 14 August 2014 Accepted: 19 February 2015 Published online: 08 March 2015

\section{References}

1. Sansom OJ, Reed KR, Hayes AJ, Ireland H, Brinkmann H, Newton IP, et al. Loss of Apc in vivo immediately perturbs Wnt signaling, differentiation, and migration. Genes Dev. 2004;18:1385-90.

2. Korobko IV, Kabishev AA, Kiselev SL. Identification of the new protein kinase specifically transcribed in mouse tumors with high metastatic potential. Dokl Akad Nauk. 1997;354(4):554-6.

3. Korobko IV, Kalinichenko SV, Korobko EV, Ninkina NN, Kiselev SL, Buchman VL. Pro-survival activity of the MAK-V protein kinase in PC12 cells. Cell Cycle. 2010;9(20):4248-9.

4. Gardner HP, Wertheim GBW, Ha SI, Copeland NG, Gilbert DJ, Jenkins NA, et al. Cloning and characterization of Hunk, a novel mammalian SNF1-related protein kinase. Genomics. 2000;63:46-59.

5. Kalinichenko SV, Itoh K, Korobko EV, Sokol SY, Buchman VL. Korobko IV identification of Nedd4 E3 ubiquitin ligase as a binding partner and regulator of MAK-V protein kinase. PLoS One. 2012;7(6):e39505. 
6. Kalinichenko SV, Vikhreva PN, Korobko IV. Interaction between MAK-V protein kinase and synaptopodin. Biochemistry (Mosc). 2011;76(2):196-201.

7. Korobko IV, Korobko EV, Kiselev SL. The MAK-V protein kinase regulates endocytosis in mouse. Mol Gen Genet. 2000;264(4):411-8.

8. Quintela-Fandino M, Arpaia E, Brenner D, Goh T, Yeung FA, Blaser H. HUNK suppresses metastasis of basal type breast cancers by disrupting the interaction between PP2A and cofilin-1. Proc Natl Acad Sci U S A. 2010;107(6):2622-7.

9. Gardner HP, Belka GK, Wertheim GB, Hartman JL, Ha SI, Gimotty PA, et al. Developmental role of the SNF1-related kinase Hunk in pregnancy-induced changes in the mammary gland. Development. 2000;127(20):4493-509.

10. Saki M, Tamura K, Tsurumi Y, Tanaka Y, Koide Y, Matsuda M, et al. Renal Expression of MAK-V/Hunk in renal distal tubules and its possible involvement in proliferative suppression. Am J Physiol. 2007;292(5):F1526-36.

11. Korobko IV, Zavalishina LE, Kiselev SL, Raǐkhlin NT, Frank GA. Proteinkinase MAK-V/Hunk as a possible diagnostic and prognostic marker of human breast carcinoma. Arkh Patol. 2004;66(5):6-9.

12. Wertheim GBW, Yang TW, Pan T, Ramne A, Liu Z, Gardner HP, et al. The Snf1-related kinase, Hunk, is essential for mammary tumor metastasis. Proc Natl Acad Sci U S A. 2009;106:15855-60.

13. Yeh ES, Yang TW, Jung JJ, Gardner HP, Cardiff RD, Chodosh LA. Hunk is required for HER2/neu-induced mammary tumorigenesis. J Clin Invest. 2011;121(3):866-79.

14. Yeh ES, Belka GK, Vernon AE, Chen CC, Jung JJ. Chodosh LA Hunk negatively regulates $\mathrm{c}$-myc to promote Akt-mediated cell survival and mammary tumorigenesis induced by loss of Pten. Proc Natl Acad Sci U S A. 2013;110(15):6103-8.

15. Kibardin A, Ossipova O, Sokol SY. Metastasis associated kinase modulates Wnt signaling to regulate brain patterning and morphogenesis. Development. 2006;133(15):2845-54.

16. Tybulewicz VL, Crawford CE, Jackson PK, Bronson RT, Mulligan RC. Neonatal lethality and lymphopenia in mice with a homozygous disruption of the c-abl proto-oncogene. Cell. 1991;65:1153-63.

17. Ninkina N, Papachroni K, Robertson DC, Schmidt O, Delaney L, O'Neil F, et al. Neurons expressing the highest levels of $\mathrm{Y}$-synuclein are unaffected by targeted inactivation of the gene. Mol Cel Biology. 2003;23:8233-45.

18. Buchman $V L$, Luke $C$, Borthwick EB, Ninkina N. Organisation of the mouse Ruk locus and expression of isoforms in mouse tissues. Gene. 2002;295:13-7.

19. Livak KJ, Schmittgen TD. Analysis of relative gene expression data using real-time quantitative $P C R$ and the 2(-delta delta $C(T))$ method. Methods. 2001:25:402-8.

20. Reed KR, Athineos D, Meniel VS, Wilkins JA, Ridgway RA, Burke ZD, et al. B-catenin deficiency, but not Myc deletion, suppresses the immediate phenotypes of Apc loss in the liver. Proc Natl Acad Sci U S A. 2008;105(48):18919-23s.

21. Xu B, Chen C, Chen H, Zheng SG, Bringas Jr P, et al. Smad1 and its target gene Wif1 coordinate BMP and Wnt signaling activities to regulate fetal lung development. Development. 2011;138(5):925-35

22. Dao DY, Yang X, Chen D, Zuscik M, O'Keefe RJ. Axin1 andAxin2 are regulated by TGF- and mediate cross-talk between TGF- and Wnt signaling pathways. Ann N Y Acad Sci. 2007;1116:82-99.

\section{Submit your next manuscript to BioMed Central and take full advantage of:}

- Convenient online submission

- Thorough peer review

- No space constraints or color figure charges

- Immediate publication on acceptance

- Inclusion in PubMed, CAS, Scopus and Google Scholar

- Research which is freely available for redistribution 